\title{
Tempo de tela, sintomas depressivos e sono: o ensino superior remoto na Covid-19
}

\author{
Screen time, depressive symptoms and sleep: remote higher education at Covid-19
}

Tiempo frente a la pantalla, síntomas depresivos y sueño: educación superior remota en Covid-19

Renata Maria Silva Santos ${ }^{1 *}$, Isabela Vitória Alves Rodrigues ${ }^{2}$, Arthur Paixão Silva Santos ${ }^{1}$, Athos Paixão Silva-Santos ${ }^{3}$, Níbia Mariana Eleutério².

\section{RESUMO}

Objetivo: Investigar a prevalência de sintomas depressivos e sua correlação com hábitos de sono e exposição excessiva à tempo de tela, em estudantes do ensino superior. Métodos: Trata-se de estudo clínico transversal, conduzido em uma faculdade privada de Belo Horizonte/Minas Gerais. Após aprovação ética, 50 estudantes foram recrutados por meio de plataformas digitais. Foram realizadas avaliações mediante aplicação de questionários padronizados por meio de chamadas telefônicas de vídeo. Os indivíduos foram avaliados quanto aos desf echos de depressão e sono. Foram coletados dados de hábitos de vida e exposição à dispositivos eletrônicos baseados em tela. Resultados: Dentre os resultados obtidos, $100 \%$ dos indivíduos relataram exposição excessiva à tempo de tela, 98\% apresentavam algum grau de sintomas depressivos e 85,7\% distúrbios de sono. Conclusão: Prevalência significativa dos sintomas depressivos na amostra investigada. São necessários estudos mais robustos, que avaliem se os sintomas depressivos e problemas de sono persistem, após o término do isolamento e retorno às atividades acadêmicas presenciais.

Palavras-chave: Depressão, Sono, Tempo de tela, Ensino superior, Pandemia.

\section{ABSTRACT}

Objective: To investigate the prevalence of depressive symptoms and their correlation with sleep habits and excessive exposure to screen time in higher education students. Methods: This is a cross-sectional clinical study conducted at a private college in Belo Horizonte/Minas Gerais. After ethical approval, 50 students were recruited through digital platforms. Assessments were carried out through the application of standardized questionnaires through video phone calls. Subjects were assessed for depression and sleep outcomes. Data on lifestyle habits and exposure to screen-based electronic devices were collected. Results: Among the results obtained, $100 \%$ of the individuals reported excessive exposure to screen time, $98 \%$ had some degree of depressive symptoms and $85.7 \%$ had sleep disorders. Conclusion: Significant prevalence of depressive symptoms in the investigated sample. More robust studies are needed to assess whether depressive symptoms and sleep problems persist after the end of isolation and return to face-to-face academic activities.

Key words: Depression, Sleep, Screen time, Education higher, Pandemic.

\section{RESUMEN}

Objetivo: Investigar la prevalencia de síntomas depresivos y su correlación con los hábitos de sueño y exposición excesiva al tiempo de pantalla en estudiantes de educación superior. Métodos: Este es un estudio clínico transversal realizado en una universidad privada en Belo Horizonte/Minas Gerais. Luego de la

\footnotetext{
1 Universidade Federal de Minas Gerais (UFMG), Belo Horizonte - MG *renatamaria1381@hotmail.com

2 Faculdade de Minas (FAMINAS-BH), Belo Horizonte - MG.

3 Universidade Estadual de Campinas (UNICAMP), Campinas - SP
} 
aprobación ética, 50 estudiantes fueron reclutados a través de plataformas digitales. Las evaluaciones se realizaron mediante la aplicación de cuestionarios estandarizados a través de videollamadas. Los sujetos fueron evaluados para la depresión y los resultados del sueño. Se recogieron datos sobre hábitos de vida y exposición a dispositivos electrónicos basados en pantallas. Resultados: Entre los resultados obtenidos, el $100 \%$ de los individuos reportaron exposición excesiva al tiempo de pantalla, el $98 \%$ presentó algún grado de sintomatología depresiva y el $85,7 \%$ presentó trastornos del sueño. Conclusión: Prevalencia significativa de síntomas depresivos en la muestra investigada. Se necesitan estudios más robustos para evaluar si los síntomas depresivos y los problemas de sueño persisten después de terminar el aislamiento y regresar a las actividades académicas presenciales.

Palabras clave: Depresión, Sueño, Tiempo de pantalla, Educación superior, Pandemia.

\section{INTRODUÇÃO}

As pandemias são manif estações coletivas de doenças de rápida disseminação com proporções mundiais, resultando em extenso número de mortes, além de interferências no funcionamento normal da cultura social e da economia dos países af etados (AKIN L e GÖZEL MG, 2020). Atualmente, o mundo vivencia a doença ocasionada pelo vírus 2019-SARS-CoV-2 (novo coronavírus), mundialmente conhecida como COVID-19, responsável por causar a síndrome respiratória aguda grave. Os primeiros relatos de infecção ocorreram em dezembro de 2019, em Wuhan, província de Hubei na China. A rápida contaminação das pessoas disseminou a doença em vários países, sendo caracterizada pela Organização Mundial de Saúde (OMS) como uma pandemia (CHAKRABORTY I e MAITY P, 2020).

Com a pandemia, tornaram-se necessárias medidas de segurança em saúde coletiva e individual, como tentativa de contenção da propagação, como por exemplo, novos investimentos em medidas de higiene, educação em saúde e adesão ao isolamento ou distanciamento social (BANERJEE D e RAI M, 2020). O isolamento envolveu o fechamento de locais públicos como shoppings e escolas, mantendo em funcionamento com restrições apenas os estabelecimentos prestadores de serviços essenciais como venda de alimentos, medicamentos ou serviços hospitalares (BEZERRA KP, et al., 2020). A partir dessas medidas de contenção da infecção, foram necessárias novas adaptações em relação à rotina mundial e manutenção do rendimento socioeconômico, aumentando assim alternativas de trabalho e Educação À Distância (EAD) (DIAS E e PINTO FCF, 2020).

O ensino remoto foi instituído no país por meio de um processo gradual de aderência das instituições às plataformas virtuais de transmissão dos conteúdos, de modo que não impactasse o rendimento acadêmico dos estudantes (BEZERRA KP, et al., 2020). Contudo, no decorrer desse processo foram evidenciadas dificuldades de interface instituição e aluno, bem como aluno e aprendizado. A desigualdade social latente no país assume implicação importante no novo contexto educacional. O que se tem observado transita entre a falta de um ambiente propício para estudos em casa, falta de recursos de internet, dificuldade de se instituir uma rotina que inclua tempo para todas as atividades acadêmicas, além do estresse causado pela mudança no formato do ensino (DIAS E e PINTO FCF, 2020).

De acordo com Banerjee D e Raim M (2020) a solidão e o estresse são de grande influência para o aparecimento de sintomas de doenças psiquiátricas. Em um contexto de isolamento social, de adaptações para o ensino à distância e dificuldades emocionais frente a uma doença ameaçadora, o cenário torna-se propício ao surgimento de problemas relacionados à saúde e bem-estar psíquico dos indivíduos. Doenças psiquiátricas relacionadas à responsiva do sistema nervoso central a situações cotidianas são comuns, segundo a Organização Pan-Americana de Saúde (OPAS), atualmente no cenário mundial. Uma dessas doenças é o Transtorno Depressivo Maior (TDM), uma condição de caráter multifatorial, segundo o Manual Diagnóstico e Estatístico de Transtornos Mentais (DSM-V), que já é considerado pela OMS como um problema de saúde pública (AMERICAN PSYCHIATRIC ASSOCIATION (APA), 2014; OPAS, 2018). 
O TDM apresenta alguns sintomas principais que incluem a ausência de afeto, falta de autocuidado, sentimento de culpa e inutilidade, perda de sono e apetite, sensação de desânimo, desinteresse, pessimismo, crises de choro, episódios de tristeza profunda e ansiedade (LIMA SO, et al., 2019). Na maioria das vezes, os sintomas depressivos manifestam-se no início da vida adulta, principalmente nos períodos de transição, onde são necessárias várias adaptações na rotina, novas responsabilidades, pressões psicológicas, mudança no núcleo de convivência social e muitas vezes associado como af astamento familiar. Essas situações podem servir como gatilho emocional para desencadear sintomas depressivos, podendo evoluir para novos casos de depressão (APA, 2014; OPAS, 2018).

Os sintomas depressivos podem afetar a capacidade cognitiva, psicomotora e comportamental do indivíduo, apresentando evoluções variadas, sendo que a condição de cronicidade é um fator influente no desenvolvimento de outros transtornos (LOUIS ED, et al., 2018; APA, 2014). Em casos de evolução da condição, podem aparecer sintomas mais preocupantes como a propensão a tentativas de autoextermínio, importante causa de morte entre indivíduos dos 15 aos 29 anos (BARROSO SM, et al., 2019). Os sintomas depressivos apresentam ainda uma associação complexa com os hábitos de sono, podendo desencadear excesso ou falta de sono, além de distúrbios do sono (NEYER M, et al., 2013).

Em se tratando de necessidade humana os determinantes de qualidade do sono transcendem à emergência fisiológica, apresentando-se como um problema crescente da sociedade urbana atual (GRANDNER MA, 2020). Distúrbios do sono af etam cerca de um quarto da população mundial, sendo que pessoas que sofrem com problemas de sono são mais propensas a desenvolver condições como transtorno de ansiedade generalizada, ideação suicida e principalmente depressão (FANG $\mathrm{H}$, et al., 2019). A presença de distúrbio de sono é sintoma comum no TDM, sendo inclusive utilizado como um dos critérios para diagnóstico de depressão e indivíduos não deprimidos com insônia, apresentam maior risco de desenvolver depressão em comparação com aqueles sem alterações do sono (NEYER M, et al., 2013).

Na população de estudantes universitários é comum a presença de alterações dos hábitos de sono que incluem dificuldade em adormecer, sonolência diurna e sono de qualidade ruim (BECKER SP, et al., 2018). As alterações dos hábitos de sono e a privação do sono entre estudantes universitários têm sido associadas à disfuncionalidades sociais e psíquicas como irritabilidade, dificuldades acadêmicas, abuso de substâncias, problemas de saúde mental, ideação suicida, além de problemas de saúde física (LI L, et al., 2018; NEYER M, et al., 2013; LI Y, et al., 2020).

Outro fator muito influente no surgimento de sintomas depressivos, principalmente quando associados à má qualidade de sono, é o tempo de tela excessivo. Tempo de tela é descrito como o tempo gasto na utilização de quaisquer dispositivos baseados em tela (WANG X, et al., 2019). O uso dos aparelhos eletrônicos atualmente tem sido cada vez mais frequente no cotidiano das pessoas. Diante de um dos piores surtos de que se tem conhecimento na história da humanidade, pessoas ao redor do mundo foram pressionadas a utilizar a tecnologia digital em ritmo e escala sem precedentes, de forma que em um momento como esse, a tecnologia digital trouxe muitos benefícios, mas ainda não estão claros os impactos do aumento dessa exposição às telas (WONG CW, et al., 2021).

Embora ainda não exista limite de tempo de tela recomendado discriminadamente para cada faixa etária, a Academia Americana de Pediatria (AAP) recomenda no máximo 2 horas de uso de aparelhos eletrônicos baseados em tela por dia, à partir de cinco anos de idade até a adolescência (REID CHASSIAKOS YL, et al., 2016).

Em países como a China e Taiwan os governos têm buscado implementar políticas com objetivo de impor limites de exposição às telas, sobretudo como meio de evitar problemas oftalmológicos em crianças e adolescentes. Na China, o uso de dispositivos eletrônicos como ferramenta educacional não deve ultrapassar a 30\% do tempo total do ensino, não mais que 20 minutos por dia para trabalhos acadêmicos de casa por meio de telas, além de proibição de telefones e tablets em salas de aula. Em Taiwan foi incluído na regulamentação que proíbe menores de 18 anos de consumir bebidas alcoólicas, fumar, e usar drogas, também o uso de dispositivos digitais por tempo excessivo, entretanto não está definido quanto tempo seria adequado (WONG CW, et al., 2021). 
Sabendo-se que sintomas depressivos são prevalentes entre jovens e adultos e que a exposição à tempo de tela excessivo é também muito comum nessa fase da vida, é possível estarmos diante de um gatilho para agravamento da situação, uma vez que também as atividades acadêmicas, no presente momento, estão dependentes de aparelhos eletrônicos de tela (WANG X, et al., 2019; FENG Q, et al., 2014; WU X, et al., 2016).

Considerando a presença de sintomas depressivos como processo incapacitante e que as alterações do sono, e o aumento do tempo de tela, ocasionados pela pandemia, podem aumentar a suscetibilidade dos estudantes ao aparecimento desses sintomas, este estudo investigou a prevalência de sintomas depressivos e sua correlação com hábitos de sono e tempo de tela, em estudantes do ensino superior, em formato remoto, durante a pandemia por COVID-19.

\section{MÉTODOS}

\section{Desenho do estudo e aspectos éticos}

Este é um estudo clínico transversal, aprovado pelo Comitê de Ética em Pesquisa sob o número de parecer 4.183.466 e CAAE 35050720.0.0000.8107. A população amostral foi composta por alunos de faculdade privada da cidade de Belo Horizonte, Minas Gerais, Brasil. Foram recrutados estudantes maiores de 18 anos de idade, de ambos os sexos. Devido ao cenário atual de isolamento social, os indivíduos foram convidados a participar da pesquisa por meio das plataformas virtuais às quais se incluíram grupos de estudantes formados nos aplicativos WhatsApp, Instagram e Facebook, bem como por contatos telefônicos. Aqueles indivíduos que manifestaram desejo em participar foram convidados a ler e assinar digitalmente o Termo de Consentimento Livre e Esclarecido (TCLE) e orientados quanto aos procedimentos de aplicação de questionários e os riscos inerentes.

\section{Procedimentos}

A aplicação dos questionários para coleta dos dados foi realizada mediante chamadas telefônicas de áudio e vídeo, de 10/08/2020 à 11/09/2020, período em que estavam participando das aulas em formato online, por meio de plataformas para disponibilização de conteúdos e realização de reuniões. Foram incluídos os seguintes dados dos participantes: idade, sexo, área do curso superior em andamento, hábitos de vida (etilismo, tabagismo e prática de atividade física) e tempo total de atividades baseadas em tela. Os participantes foram caracterizados quanto à presença e gravidade de sintomas depressivos, por meio do Inventário de Depressão de Beck (BDI) uma escala que avalia a presença e intensidade dos sintomas depressivos, composta por 21 itens de 5 afirmativas em cada um deles (SCHUCH FB, et al., 2020).

A presença de distúrbios do sono e sonolência excessiva diurna foi avaliada por meio dos questionários Índice de Qualidade do Sono de Pittsburgh (PSQI) e Escala de Sonolência Epworth (ESE). O PSQI é utilizado para avaliar os hábitos/qualidade de sono do indivíduo, referentes ao último mês, validado no Brasil em 2011, já a ESE é utilizada para classificar situações cotidianas associadas à sonolência, que evidenciam desde sono normal, até uma sonolência possivelmente patológica (KENDZERSKA TB, et al., 2014; PASSOS MH, et al., 2017). Como ainda não está estabelecido um tempo de tela adequado à cada ciclo do desenvolvimento humano, este estudo tomou como base as diretrizes da AAP, que recomenda no máximo 2 horas de uso de aparelhos eletrônicos baseados em tela por dia, a partir de cinco anos de idade até a adolescência (REID CHASSIAKOS YL, et al., 2016). Os participantes do estudo estavam recebendo as aulas em formato online, de modo que foi considerado excessivo o tempo de tela superior a cinco horas por dia, às quais se incluiriam três horas de conteúdo acadêmico habitual da instituição e o restante para outros fins.

\section{Análise dos dados}

Foi realizada uma análise descritiva das variáveis por meio de tabelas de distribuição de frequências, medidas de tendência central (média), variabilidade (desvio-padrão). Posteriormente foi verificada a associação entre as variáveis estudadas pela variável que indica o nível de depressão dos participantes. Para essa comparação foi utilizado o teste Qui-quadrado de Pearson ou teste exato de Fisher para análise das variáveis categóricas. Em todas as análises foi considerado um nível de significância de $5 \%$, valor de p<0,05, utilizando o software estatístico SPSS versão 22.0. 


\section{RESULTADOS}

A instituição de ensino superior privada em que foi desenvolvida a pesquisa possui cerca de 2.000 alunos. A população amostral do estudo foi constituída por 50 alunos, em sua totalidade matriculados em cursos da área da saúde (medicina, biomedicina e farmácia), com idade média de 25 anos, sendo a maioria 64\%, do sexo feminino. Com relação aos hábitos de vida, $100 \%$ da amostra não consumia bebidas alc oólicas, $98 \%$ não fumava e $70 \%$ da não praticava atividades físicas. No que se refere ao tempo de tela, $100 \%$ dos participantes relataram tempo de tela excessivo.

Na Tabela 1, foram compilados os dados obtidos por meio dos questionários utilizados para a pesquisa, que compreendem o PSQI, o BDI e a ESE. Os escores referentes à presença ou ausência do distúrbio pesquisado pelo questionário foram classificados como sim e não, respectivamente, permitindo obtenção da porcentagem da amostra, sendo que para o BDI foi possível também estratificar por gravidade dos sintomas.

Tabela 1 - Resultados dos questionários padronizados para sintomas depressivos e sono.

\begin{tabular}{|c|c|c|c|}
\hline Variáveis & Frequência & Porcentagem & Gravidade \\
\hline \multirow{2}{*}{$B D I^{*}$} & \multirow{2}{*}{ 49-Sim } & \multirow{2}{*}{$98 \%$} & Mínimo= 40,8\% \\
\hline & & & Leve $=30,6 \%$ \\
\hline Sintomas depressivos & & $2 \%$ & Moderado $/$ severo $=28,6 \%$ \\
\hline \multirow{2}{*}{$\mathrm{ESE}^{*}$} & 32- Sim & $64 \%-\operatorname{Sim}$ & \multirow{2}{*}{$N A^{*}$} \\
\hline & 18- Não & 36\%- Não & \\
\hline \multirow{2}{*}{ PSQI* } & 44- Sim & $88 \%-S i m$ & \multirow{2}{*}{$N A^{*}$} \\
\hline & 6- Não & 12\%-Não & \\
\hline
\end{tabular}

Legenda: *Índice de Qualidade do Sono de Pittsburgh (PSQI); *Inventário de Depressão de Beck (BDI); ${ }^{*}$ Escala de Sonolência Epworth (ESE); *Não se Aplica (NA).

Fonte: Santos RMS, et al., 2022.

Para realização da associação entre os sintomas depressivos e os demais aspectos avaliados neste estudo, foi realizada uma associação entre a presença ou não dos distúrbios do sono, da sonolência excessiva diurna, prática de atividade física e tempo de tela e a gravidade dos sintomas depressivos. Foi utilizado como estatisticamente significativo o valor de $p<0,05$, evidenciando ausência de correlação entre as variáveis analisadas, como observado na Tabela 2.

Tabela 2 - Associação entre os níveis de sintomas depressivos e as demais variáveis do estudo.

\begin{tabular}{lcccc}
\hline Sintomas depressivos Variáveis & Grau Mínimo & Grau Leve & $\begin{array}{c}\text { Grau } \\
\text { Moderado/Severo }\end{array}$ & Valor-p \\
\hline Sonolência Diurna & $\mathbf{N = 2 0}$ & $\mathbf{N = 1 5}$ & $\mathbf{N = 1 4}$ & \\
Distúrbios do sono & $10(50 \%)$ & $12(80 \%)$ & $10(71,4 \%)$ & $0,184^{*}$ \\
Tempo de tela Excessivo & $18(90 \%)$ & $13(86,7 \%)$ & $12(85,7 \%)$ & $1,000^{*}$ \\
\hline Atividade física & $20(100 \%)$ & $15(100 \%)$ & $14(100 \%)$ & - \\
\hline Sim & & & & \\
Não & $5(25 \%)$ & $5(33,3 \%)$ & $4(28,6 \%)$ & $0,922^{*}$ \\
\hline
\end{tabular}

Legenda: *Teste exato de Fisher $(n=49)$.

Fonte: Santos RMS, et al., 2022. 


\section{DISCUSSÃo}

O grupo amostral final de 50 alunos foi menor que o estimado pelo estudo, tendo em vista o número de alunos da instituição de ensino superior em que foi realizada a pesquisa. A amostra relativamente pequena, considerando estudos dessa natureza, se justifica pelo cenário de isolamento social, em que há maior dificuldade em mobilizar os universitários à distância. Os dados demog ráficos e a caracterização da área de estudo dos participantes da presente pesquisa estão compatíveis com outros levantamentos e contribuem para o escopo da literatura. Segundo estudo semelhante realizado com 383 acadêmicos da área da saúde, a maioria da amostra foi composta por mulheres, $70,3 \%$ e não foi detectada associação entre depressão e gênero. Observa-se pelos escores do BDI, que os sintomas depressivos entre estudantes da área da saúde têm se mostrado superior à outras populações de idade correspondente (LIMA SO, et al., 2019; BHASKARA NV, et al., 2020).

Diante desses achados é importante aventar a necessidade de of erta do apoio psicológico institucional para essa população, bem como a disseminação do conhecimento acerca do problema (BRESOLIN JZ, et al., 2020). Da mesma forma, entender a condição psicológica dos estudantes pode fornecer um norte para instituições que desejem investir em apoio, bem como fomentar as bases para políticas públicas nesse sentido. Sob esse prisma, outros estudos também concluíram que a interrupção das aulas presenciais, provocada pela pandemia, mostrou desafios e oferece oportunidades de avaliação de estratégias para melhorias na educação (CHATURVEDI K, et al., 2021).

$\mathrm{Na}$ amostra avaliada, os indivíduos que apresentaram grau moderado a severo de depressão $(71,4 \%)$, $100 \%$ deles estavam expostos a tempo excessivo de tela, aliado a isto, $85,7 \%$ apresentaram distúrbios de sono. Em relação ao consumo excessivo de tempo de tela, Carone CMM, et al. (2020) relatam em seu estudo prevalência de $30 \%$, ao passo que Lourenço CLM, et al. (2016) já haviam observado perc entual maior, $83,7 \%$. No presente estudo $100 \%$ dos indivíduos reportaram exposição excessiva às telas. No entanto, este cenário pode ser explicado devido ao afastamento social, em que a utilização de aparelhos eletrônicos como mecanismo compensatório automático se agravou ainda mais, pela necessidade latente do uso de ambientes virtuais para muitas atividades de vida diária como trabalho e estudo. Diante de contexto pandêmico era esperado maior utilização de dispositivos eletrônicos baseados em telas, entretanto é importante salientar que esse uso já vinha crescendo (WANG X, et al., 2019; DIAS E e PINTO FCF, 2020).

A avaliação dos hábitos de sono também encontrou consonância na literatura, uma vez que estudos já mostraram que os distúrbios de sono estão presentes em indivíduos que tem maior predisposição a desenvolver depressão, podendo ser considerado um dos sintomas iniciais do transtorno (NEYER M, et al., 2013). Os instrumentos utilizados neste estudo avaliaram os indivíduos em um curso temporal que possibilita o relato da presença de sintomas depressivos e hábitos de sono percebidos nas semanas anteriores à entrevista. Sendo assim, é possível que esses dados não reflitam fidedignamente a realidade dessa população, uma vez que podem estar enviesados pela dificuldade de recordar dados, ou impactados pela própria condição estressante de pandemia.

O contexto de bloqueio afetou de diferentes formas a rotina da população ao redor do mundo. Estudo realizado na população geral de Singapura avaliou os comportamentos de movimento das pessoas em várias faixas etárias. Encontraram um aumento de duração do sono eficiente durante o bloqueio, concluindo que a atividade física foi mais gravemente af etada do que o sono (ONG JL, et al., 2021).

Os universitários avaliados nesta pesquisa apresentaram perfil predominantemente sedentário. Em estudos anteriores à pandemia por COVID-19, realizados em universidades públicas brasileiras, mais da metade dos participantes relataram praticar atividades físicas (CARONE CMM, et al., 2020; LOURENÇO CLM, et al., 2016). É importante salientar que existem diferenças na rotina de universitários de instituições de ensino privadas e públicas, de modo geral, estudantes de instituições privadas de ensino superior precisam conciliar trabalho e estudo, o que diminui as oportunidades de dedicação a atividades físicas (MORETTI FA e HUBNER MMC, 2017). 
Entretanto, os dados capturados com essa pesquisa não são suficientes para entender se existe de fato essa diferença entre universitários de instituições privadas e públicas, tanto pelo número reduzido da amostra, quanto pela falta de dados anteriores ao período de isolamento. A falta de atividade física aliada ao isolamento social, o ensino remoto com privação do contato presencial entre pares, pode também estar contribuindo para aparecimento ou agravamento de sintomas depressivos em universitários.

A disseminação da COVID-19 e o fechamento forçado de instituições de ensino ao redor do mundo, foi desafiador para as universidades no sentido de lidar com uma crise. A mudança maciça e repentina para o ensino remoto acelerou o desenvolvimento dos ambientes online, para manter um aprendizado eficaz, mesmo de forma virtual. Contudo estudos mostraram que alunos e professores encontraram dificuldades para organizar a carga de trabalho, a comunicação e o uso das tecnologias, além do aumento do tempo gasto nas plataformas online, comparado às condições anteriores a pandemia. Embora este formato de ensino possa fornecer maior flexibilidade, existem os distratores ambientais do estudo em casa com a família e os problemas de conexão com a internet, que podem ser uma barreira. Do ponto de vista institucional, estudos como este podem contribuir para refinar o apoio acadêmico e social ao universitário (PROKES C e HOUSEL J, 2021; DOST S, et al., 2020).

A influência da pandemia por Covid-19 e todas as alterações decorrentes desta, como o isolamento social e a necessidade de ensino remoto, podem ter sido, nesse estudo, fatores influentes para a alta prevalência de sintomas depressivos e problemas de sono, autorrelatados em universitários de instituição privada de ensino superior. Mesmo em período de isolamento social, no qual se espera encontrar nos resultados tempo maior dedicado à atividade de dormir, esses estudantes apresentaram números significativos de problemas relacionados ao sono, sobretudo quando adicionado à necessidade de maior tempo de tela. É importante mencionar a limitação inerente ao tamanho reduzido da amostra do estudo, que inviabiliza a generalização dos resultados. $O$ interferente ambiental do contexto pandêmico adiciona fragilidade aos dados capturados com a presente pesquisa, impossibilitando a estratificação do tempo de tela dedicado aos estudos e a outros fins, como interação social ou mesmo entretenimento, em condição de isolamento.

\section{CONCLUSÃO}

Este estudo encontrou resultados importantes da exposição excessiva às telas. Os impactos do tempo de tela na função af etiva e hábitos de sono dos jovens, foram agravados, em parte, pela condição de isolamento social, imposta pela pandemia Covid-19. Embora os dados sejam referentes à uma pequena amostra populacional, é possível que o modelo de estudo aqui conduzido possa contribuir como parte de protocolo de monitoramento da saúde mental de universitários. Entretanto, serão necessários estudos complementares, mais robustos, que avaliem se os sintomas depressivos e problemas de sono persistem após o término do isolamento e retorno às atividades acadêmicas presenciais, bem como à rotina anterior de exposição às telas .

\section{REFERÊNCIAS}

1. AKIN L, GÖZEL MG. Understanding dynamics of pandemic. Turkish Journal of Medical Sciences, 2020;50(SI-1):515519.

2. AMERICAN PSYCHIATRIC ASSOCIATION (APA). Manual diagnóstico e estatístico de transtornos mentais: DSM-V. $5^{\mathrm{a}}$ ed. Porto Alegre: Artmed, 2014.

3. BANERJEE D, RAI M. Social isolation in Covid-19: The impact of loneliness. International Journal of Social Psychiatry, 2020;66(6): 525-527.

4. BECKER SP, et al. Sleep in a large, multi-university sample of college students: sleep problem prevalence, sex differences, and mental health correlates. Sleep Health, 2018;4(2): 174-181.

5. BEZERRA KP, et al. Ensino remoto em universidades públicas estaduais: o futuro que se faz presente. Research, Society and Development, 2020;9(9): e359997226.

6. BHASKARA NV, et al. The effect of social media use on the mental well-being of medical students in Bangalore, Karnataka. Journal of Family Medicine and Primary Care, 2020; 9(11):5731-5735.

7. BRESOLIN JZ, et al. Sintomas depressivos em estudantes universitários da área da saúde. Revista Latino-Americana de Enfermagem, 2020;28:e3239. 
8. CARONE CMM, et al. Fatores associados a distúrbios do sono em estudantes universitários. Cadernos de Saúde Pública, 2020;36(3): e00074919.

9. CHAKRABORTYI, MAITY P. COVID-19 outbreak: Migration, effects on society, global environment, and prevention. Science of the Total Environment, 2020;728: 138882.

10. CHATURVEDI K, et al. COVID-19 and its impact on education, social life and mental health of students: A survey. Children and Youth Services Review, 2021;121: 105866.

11. DIAS E, PINTO FCF. A Educação e a Covid-19. Ensaio: avaliação em políticas públicas em educação,2020;28(108): 545-554.

12. DOST S, et al. Perceptions of medical students towards online teaching during the COVID-19 pandemic: a national cross-sectional survey of 2721 UK medical students. BMJ Open, 2020;10(11): e042378.

13. FANG H, et al. Depression in sleep disturbance: A review on a bidirectional relationship, mechanisms and treatment. Journal of Cellular and Molecular Medicine, 2019;23(4):2324-2332.

14. FENG Q, et al. Associations of Physical Activity, Screen Time with Depression, Anxiety and Sleep Quality among Chinese College Freshmen. Journal Plos One, 2014;9(6): e0100914.

15. GRANDNER MA. The Cost of Sleep Lost: Implications for Health, Performance, and the Bottom Line. American joumal of health promotion, 2018;32(7): 1629-1634.

16. GRANDNER MA. Sleep, Health, and Society. Sleep Medicine Clinics, 2020;15(2):319-340.

17. KENDZERSKA TB, et al. Evaluation of the measurement properties of the Epworth sleepiness scale: a systematic review. Sleep Medicine Reviews, 2014; 18(4): 321-331.

18. LI L, et al. Prevalence of sleep disturbances in Chinese university students: a comprehensive meta-analysis. Joumal of sleep research, 2018;27(3): e12648.

19. LIMA SO, et al. Prevalência da Depressão nos Acadêmicos da Área de Saúde. Psicologia: Ciência e Profissão, 2019; (39): $1-14$

20. LI Y, et al. Prevalence and correlates of poor sleep quality among college students: a cross-sectional survey. Health Quality of Life Outcomes, 2020; 18(1):210.

21. LOUIS ED, et al. Merrit's neurology. $13^{\mathrm{a}} \mathrm{ed}$. Rio de janeiro: Guanabara Koogan, 2018;461p.

22. LOURENÇO CLM, et al. Comportamento sedentário em estudantes Universitários. Revista Brasileira de Atividade Física e Saúde, 2016;21(1):67-77

23. MORETTI FA, HUBNER MMC. O estresse e a máquina de moer alunos do ensino superior: vamos repensar nossa política educacional?. Revista psicopedagogia, 2017;34(105):258-267.

24. MUKHTAR K, et al. Advantages, Limitations and Recommendations for online learning during COVID -19 pandemic era. Pakistan journal of medical sciences, 2020;36(COVID19-S4): S27-S31.

25. NYER M, et al. Relationship between sleep disturbance and depression, anxiety, and functioning in college students. Depression and Anxiety, 2013;30(9): 873-80.

26. ONG JL, et al. COVID-19-related mobility reduction: heterogenous effects on sleep and physical activity rhythms. Sleep. 2021;44(2): zsaa179.

27. ORGANIZAÇÃO PAN AMERICANA DE SAÚDE (OPAS). Folha informativa- depressão. 2018. Disponível em https://www.paho.org/pt/topicos/depressao. Acessado em: 8 de setembro de 2020.

28. PASSOS MH, et al. Reliability and validity of the Brazilian version of the Pittsburgh Sleep Quality Index in adolescents. Jornal de Pediatria, 2017;93(2): 200-206.

29. PROKES C, HOUSEL J. Community College Student Perceptions of Remote Learning Shifts Due to COVID-19. TechTrends, 2021;6:1-13.

30. REID CHASSIAKOS YL, et al. Children and adolescents and digital media. Pediatrics, 2016; 138: e2016-2593.

31. SCHUCH FB, et al. Associations of moderate to vigorous physical activity and sedentary behavior with depressive and anxiety sym ptoms in self-isolating people during the COVID-19 pandemic: A cross-sectional survey in Brazil. Psychiatry Research, 2020;292: 113339.

32. WANG X, et al. The associations between screen timebased sedentary behavior and depression: a systematic review and meta-analysis. BMC Public Health, 2019; 19: 1524.

33. WONG CW, et al. Digital Screen Time During the COVID-19 Pandemic: Risk for a Further Myopia Boom?. American Journal of Ophthalmology, 2021;223:333-337.

34. WU X, et al. Impact of screen time on mental health problems progression in youth: a 1 -year follow-up study. BMJ Open, 2016; 6: e011533. 